\title{
Central and peripheral neurological involvement in monoclonal gammopathies of undetermined significance ${ }^{*}$
}

\author{
Edvina Galiè $^{1 \#}$, Maria Luisa Dell’Acqua², Marta Maschio ${ }^{1}$, Tatiana Koudriavtseva ${ }^{1}$, \\ Emidio De Marco ${ }^{3}$, Bruno Jandolo ${ }^{1}$ \\ ${ }^{1}$ Division of Neurology, Regina Elena National CancerInstitute, Roma, Italy \\ ${ }^{2}$ Division of Neurology, Nuovo Ospedale Civile Sant'Agostino Estens, Modena, Italy \\ ${ }^{3}$ Division of Radiology, Libera Università Campus Biomedico, Roma, Italy \\ Email: "
}

Received 8 May 2013; revised 8 July 2013; accepted 8 August 2013

Copyright (c) 2013 Edvina Galiè et al. This is an open access article distributed under the Creative Commons Attribution License, which permits unrestricted use, distribution, and reproduction in any medium, provided the original work is properly cited.

\begin{abstract}
Several studies have suggested a pathogenetic role of paraproteinaemias in PNS damage. Over the few last years, the presence of symptomatic or subclinical PNS lesions in CNS diseases like multiple sclerosis has been described. On the other hand, CNS demyelinating lesions and cervical atrophy have been reported in patients affected by chronic inflammatory demyelinating polyradiculoneuropathy (CIDP). Very few cases of MGUS associated with CNS disease alone or with both CNS and PNS disease have been reported. Since 1999, we have been studying 16 patients $(8 \mathrm{M}, 8 \mathrm{~F})$, with a mean age $60.2 \pm 13.4$, affected by MGUS associated with symptomatic neurological central and/or peripheral diseases. Patients affected with lymphomas, lupus erithematosus and other immunological diseases were excluded. Involvement of both PNS and CNS was not associated to a particular type of paraproteinemia: monoclonal IgM were found in 8 patients; monoclonal IgG in 6 patients and monoclonal IgA in 1 patient and Ig $\lambda$ in 1 patient. High antinervous system autoantibodies were found in 10/16 patients and antiMAG antibodies were detected in patients with paraproteinemic demyelinating neuropathy (PDN). High reactivity anti-nervous system might support the hypothesis of a pathogenetic role of MGUS in these neurological diseases. Nevertheless, at present, we cannot exclude that there is only a circumstantial association between MGUS and neurological damages, particularly concerning CNS.
\end{abstract}

Keywords: Multiple Sclerosis (MS); Monoclonal Gammopathies of Undetermined Significance (MGUS); Peripheral Nervous System (PNS) Involvement; Central

*Competing Interests: There are no Competing Interests.

\#Corresponding author.
Nervous System (CNS) Involvement

\section{INTRODUCTION}

While the association of monoclonal gammopathies of undetermined significance (MGUS) with peripheral nervous system (PNS) involvement has been widely documented [1-8], the involvement of the central nervous system (CNS) in the presence of MGUS has seldom been described [9-13].

Several studies have suggested a pathogenetic role of paraproteinaemias in PNS damage; 10\% of patients with an idiopathic neuropathy have an associated monoclonal gammopathy, while $8 \%$ - $37 \%$ of patients with MGUS have symptomatic neuropathy where often it is the only clinical manifestation of an underlying hematologic disorder $[3,7,14]$. The monoclonal antibodies reacting with neural antigens in MGUS are more likely to belong to the IgM class although IgG is the most common class of paraproteins in patients with MGUS [4]. Very few cases of CNS disease associated with MGUS and of the coexistence of CNS and PNS involvement in patients with a paraproteinemia are described $[14,15]$. For this reason, we have studied these particular cases of patients who have referred to our institution over the last ten years, with the aim to evaluate a possible causal relation between MGUS and CNS involvement.

\section{MATERIALS AND METHODS}

A retrospective chart review was carried out on 40 patients who were under our care from 1999 to September 2009, affected with peripheral neuropathy of unknown pathogenesis (patients with diabetes, neurotoxicity, hypovitaminosis, haematological diseases were excluded) and 71 patients with suspected demyelinating disease. We evaluated those affected by MGUS. 
We identified 16 patients (8 males and 8 females) with a mean age $60.2 \pm 13.4$ and affected by MGUS. Fifteen patients underwent haematological analysis (basic haematochemical exams, immunoelectrophoresis, neurological autoantibodies such as ANA, anti-Yo, anti-Ri, anti$\mathrm{Hu}$, anti-MAG, anti-GM1, anti-GQ1b, GD1b), and cerebrospinal fluid (CSF) examination (cytochemical, intrathecal production of IgG, oligoclonal bands-OB) research, Link Index); one patient only hadahaematological screening and not a CSF exam. All patients underwent X-Ray skeletal surveys, and neurophysiological and neuroradiological investigations: electroneuromyography (ENMG), Magnetic Resonance Imaging (MRI) of the brain and spinal cord. Each patient visited our Institute at different times, and had their serial examinations at different times, each according to their disease, their symptomathology and their responses to therapy. Patients affected by lymphomas, myeloma, amyloidosis, systemic lupus erithematosus and other immunological diseases were excluded. The follow-up varies between 2 and 12 years.

\section{RESULTS}

Patient charts were divided into two groups. Group oneCentral Nervous System damage (Total 5 patients): 4 Multiple Sclerosis (MS) and 1 cerebellar atrophy. Second group-Neuroperipheric Damage (Total 11 patients with): 8 paraproteinemic demyelinating neuropathy (PDN) and 3 axonal neuropathy. This group was then divided into 2 subgroups (first: group IIa with only PNS damage (7 patients) and group IIb with PNS and CSN damage (4 patients: 2 with PDN + Parkinson diseases (PD) and 2 with PDN + Multisystemic Atrophy (MSA) (Tables 1-3).

In group I: 2 patients had MGUS type IgM and 3 patients MGUS type IgG. All of them had CSF alteration while ANA and anti-GM1 seric positivity was found in 4 patients.

In group IIa e IIb: 6 patients had MGUS type IgM, 3 MGUS type IgG, 1 MGUS type IgA and 1 Ig $\lambda$. AntiMAG seric positivity was detected in 4 cases, all of them had been affected by PDN and with MGUS type IgM; anti-GM1 autoantibodies were present in 2 cases: 1 MGUS type IgG and 1 MGUS type IgA. CSF was abnormal in 9 cases, 3 of them had also CNS involvement (2MSA e 1 PD).

Patients with PD and MSA had a typical symptomatology, that began at the same time or after the onset of the peripheral neuropathy.

\section{DISCUSSION AND CONCLUSIONS}

Several data suggest a pathogenetic role of paraproteinaemias in PNS damage. A demyelinating neuropathy can be induced in animals after systemic administration of serum or intraneuronal injection of IgM taken from patients with a demyelinating neuropathy and MGUS. Fur-

Table 1. Demographic and laboratory data of patients with CNS damage (Group I).

\begin{tabular}{cccccc}
\hline AGE & SEX & MGUS TYPE & DISEASE & AUTO-Ab & CSF \\
\hline 49 & F & IgG K & ${ }^{*}$ RR MS & ANA (1:80) & ${ }^{\circ}$ Link Index $\uparrow$ Policlonal k chains \\
63 & M & IgM $\lambda$ & ${ }^{\circ}$ PP MS & ANA (1:80) & Oligoclonal Bands \\
46 & F & IgM $\lambda$ & ${ }^{*}$ RR MS & ANA (1:320) & ${ }^{\circ} \operatorname{Link}$ Index $\uparrow+{ }^{\circ}$ IgG $\uparrow$ \\
45 & F & IgG K & ${ }^{*}$ RR MS & ${ }^{\circ 0}$ GM1(IgM:75) & ${ }^{\circ} \operatorname{Link} \operatorname{Index} \uparrow+{ }^{\circ}$ IgG $\uparrow$ \\
34 & M & IgG $\lambda$ & CerebellarAtrophy & negative & ${ }^{\circ} \operatorname{Link} \operatorname{Index} \uparrow+{ }^{\circ}$ IgG $\uparrow+$ Oligoclonal Bands \\
\hline
\end{tabular}

*RR MS: Relapsing Remitting Multiple Sclerosis; ’PP MS: Primary Progressive Multiple Sclerosis.

Table 2. Demographic and laboratory data of patients with PNS damage (Group II a).

\begin{tabular}{|c|c|c|c|c|c|}
\hline AGE & SEX & MGUS TYPE & DISEASE & AUTO-Ab & CSF \\
\hline 57 & M & $\operatorname{IgM} \lambda$ & PDN & ${ }^{\circ 00}$ MAG (1300) & albumino-cytologicdissociation \\
\hline 74 & M & $\operatorname{IgM} \mathrm{K}$ & PDN & ${ }^{\circ 00}$ MAG $(30,000)$ ANA $(1: 80)$ & albumino-cytologicdissociation \\
\hline 59 & M & IgG K & PDN & GM1 (IgM:62) & ${ }^{\circ \circ} \mathrm{IgG} \uparrow$ \\
\hline 68 & $\mathrm{~F}$ & IgG K & "S-M axonalpolyneuropathy & negative & ${ }^{\circ 0} \operatorname{IgG} \uparrow$ \\
\hline 60 & M & $\operatorname{IgA} \lambda$ & Autonomic polyneuropathy & ${ }^{\circ 00}$ GM1 (IgM:62) & notexecuted \\
\hline 63 & $\mathrm{~F}$ & $\operatorname{Ig} \lambda$ & ${ }^{* *}$ S-axonalpolyneuropathy & negative & ${ }^{\circ \circ} \operatorname{IgG} \uparrow+$ Monoclonal Band $\lambda$ \\
\hline 79 & $\mathrm{~F}$ & $\operatorname{IgM} \lambda$ & PDN & ${ }^{\circ 00}$ MAG $(13,500)$ & albumino-cytologicdissociation \\
\hline
\end{tabular}

": sensory-motor polyneuropathy; ${ }^{* *}:$ sensory polyneuropathy; $:$ Link Index $\uparrow(>0.65) ;{ }^{\circ}:$ IgG $\uparrow\left(>3.80\right.$ mg/dl); ${ }^{\circ 00}$ : normal values Ab anti-MAG antibody titers: $<1000$ BTU; Ab anti-GM1 antibody titers: <25 EU/mlL. 
Table 3. Demographic and laboratory data of patients with CNS and PNS damage (Group II b).

\begin{tabular}{|c|c|c|c|c|c|}
\hline AGE & SEX & MGUS TYPE & DISEASE & AUTO-Ab & CSF \\
\hline 62 & $\mathrm{~F}$ & IgM K & $\mathrm{PDN}+\mathrm{PD}$ & ${ }^{\circ 00}$ anti-MAG (81000) ANA (1:80) & ${ }^{\circ}$ IgG $\uparrow+$ albumino-cytologicdissociation \\
\hline 81 & M & IgM K & $\mathrm{PDN}+\mathrm{PD}$ & negative & normal \\
\hline 75 & M & IgM K & $\mathrm{PDN}+\mathrm{MSA}$ & negative & ${ }^{\circ}$ Link Index $\uparrow+{ }^{\circ}$ IgG $\uparrow+$ albumino-cytologic dissociation \\
\hline 48 & $\mathrm{~F}$ & $\operatorname{IgG} \lambda$ & $\mathrm{PDN}+\mathrm{MSA}$ & negative & 'Link Index $\uparrow+$ Oligoclonal Band IgG $\uparrow$ \\
\hline
\end{tabular}

thermore, anti-nerve autoantibodies in the serum of patients with IgMparaproteinemic neuropathy were detected and the deposition of immunoglobulin and complement in endonevrium was demonstrated by immunofluorescent staining [7]. One-half of patients with IgM-MGUS neuropathy have an illness that is associated with antibodies against a specific myelin associated glycoprotein (antiMAG) $[14,16]$. Fluri et al. [17] demonstrated that, in patients with neuropathy associated with IgM paraproteinaemia and anti-MAG antibodies, the IgM binding strength to MAG was higher for CNS myelin than for PNS myelin, even in the absence of any clinical CNS involvement. Over the last few years, the presence of symptomatic or subclinical PNS lesions in CNS diseases like multiple sclerosis has been described [18-25]. On the other hand, CNS demyelinating lesions and cervical cord atrophy have been reported in patients affected by acute or chronic inflammatory demyelinating polyradiculoneuropathy (CIDP) [26-28].

In group 2 (PNS disease), a higher frequency of IgM was confirmed (53\%), and in more than half of the cases it was found to be associated to anti-MAG. In the 3 cases of axonal damage, there was no IgM MGUS. Regarding the 4 cases in which damage of the CNS was associated to PNS disease, we emphasize that the distinctive involvement of the extrapiramidal system and the high prevalence of this co-occurrence (36\%, that is $4 / 11$ patients), compared to the prevalence of parkinsonism in the general population (1\% over 60 years of age) [29], might support the hypothesis of a link between MGUS and such disorders. However the sample size is too small to permit a true statistical conclusion.

In group 1, 4 patients met the diagnostic criteria of late onset MS, prevalent in 30\% (over 13 of our cases). This prevalence is much higher than the risk in the association between MGUS and MS (0.0065/100) [14]. These data and the research of Fluri et al. [17] may suggest a pathogenetic link between MGUS and MS of late onset.

The case of the young man with cerebellar atrophy shows that he suffered progressive cerebellar syndrome, first unilaterally, then bilaterally. A serial MRI showed only cerebellar vermis atrophy. A CSF exam showed oligoclonal bands and elevated IgG $\lambda$ monoclonal gammopathy, but no autoantibodies. Genetic analysis for
SCA was negative and seric value of vitamin E, vitamin B12 and folic acid were normal. Nerve conduction studies were normal $[7,30]$. Far from being demonstrated, we think that a pathogenetic role of IgG MGUS in this patient could be an intriguing hypothesis.

MGUS is found in approximately 1\% - 3\% [31] of people above 50 years of age and in 3\% - 5\% above 70 years.

They evolve into malignant disorders at a rate of only $1 \%$ per year. Quality of life may be compromised by an associated disease.

It is known that a bigger sample size is needed if a true statistical association exists, however, we have reported the coexistence of MGUS in particular types of CNS disease. We think that it would be useful to test the presence of MGUS in these selected patients considering that MGUS may be the precursor of more serious diseases, such as multiple myeloma, primary amyloidosis and Waldenstrommacroglobulinemia. More importantly, if larger studies confirm the association between MGUS and these diseases, further knowledge in the pathogenetic mechanism of these complex CNS diseases will be obtained.

Our data seem to confirm the pathogenetic role of MGUS (especially IgM) in some polyneuropathies, as shown by Bida et al. [32] for CIDP. Meanwhile, a pathogenetic link, and not only a chance occurrence, may be hypothesized between MGUS and late onset MS and any extrapiramidal disorders.

\section{ACKNOWLEDGEMENTS}

We thank Ms Tania Merlino for reviewing the English of this paper.

\section{REFERENCES}

[1] Joint Task Force of the EFNS and the PNS (2010) European Federation of Neurological Societies/Peripheral Nerve Society Guidelines on management of paraproteinemic demyelinating neuropathies. Report of a Joint Task Force of the European Federation of Neurological Societies and the Peripheral Nerve Society-First revision. Journal of the Peripheral Nervous System, 15, 185-195. http://dx.doi.org/10.1111/j.1529-8027.2010.00278.x

[2] Busby, M. and Donaghy, M. (2003) Chronic dysimmune 
neuropathy, a subclassification based upon the clinical features of 102 patients. Journal of Neurology, 250, 714724. http://dx.doi.org/10.1007/s00415-003-1068-2

[3] Chassande, B. and Leger, J.M. (1993) Neurological manifestations of monoclonal gammopathies. Revue du Praticien, 43, 314-316.

[4] Drappatz, J. and Batchelor, T. (2004) Neurological complications of plasma cell disorders. Clinical Lymphoma, 5, 163-171. http://dx.doi.org/10.3816/CLM.2004.n.022

[5] Ponsford, S., Willison, H., Veitch, J., Morris, R. and Thomas, P.K. (2000) Long-term clinical and neurophysiological follow-up of patients with peripheral neuropathy associated with benign monoclonal gammopathy. Muscle Nerve, 23, 164-174.

http://dx.doi.org/10.1002/(SICI)1097-4598(200002)23:2< 164::AID-MUS4>3.0.CO;2-Y

[6] Uldry, P.A., Steck, F. and Regli, F. (1984) Manifestations neurologiques des gammapathies monoclonales. Schweizerische Medizinische Wochenschrift, 114, 1678-1685.

[7] Silberman, J. and Lonial, S. (2008) Review of peripheral neuropathy in plasma cell disorders. Hematological Oncology, 26, 55-65. http://dx.doi.org/10.1002/hon.845

[8] Merkies, I.S. and Faber, C.G. (2012) Fatigue in immunemediated neuropathies. Neuromuscular Disorders, 22, 203-207.

[9] Galiè, E., Maschio, M. and Jandolo, B. (2005) Cerebellar atrophy and monoclonal gammopathy of undetermined significance: A possible correlation? Journal of Experimental \& Clinical Cancer Research, 24, 483-485.

[10] Rentzos, M., Michalopoulu, M., Gotosidis, K., Caponi, A., Bonakis, A., Kilidireas, K. and Nikolaou, C. (2004) Unusual association of multiple sclerosis with monoclonal gammopathy of undetermined significance (MGUS): Two cases. Functional Neurology, 19, 253-256.

[11] Galiè, E., Pietrangeli, A., Maschio, M., Pace, A., Vidiri, A., Carosi, M. and Jandolo, B. (2003) Demyelinating disease in monoclonal gammopathy of undeterminated significance. Journal of Experimental \& Clinical Cancer Research, 22, 337-339.

[12] Trèfouret, S., Azulay, J.-Ph, Pouget, J., Boucraut, J. and Serratrice, G. (1996) Sclerose en plaques tardive et gammapathie monoclonal sérique: Association fortuite? Revista de Neurología, 152, 554-556.

[13] Cai, Z., Blumberg, P.C., Koblar, S.A., Kathy, C., Manavis, J., Ghabriel, M.N. and Thompson, P.D. (2004) Peripheral nervous system and central nervous system pathology in rapidly progressive lower motor neuron syndrome with immunoglobulin M anti GM1 ganglioside antibody. Journal of the Peripheral Nervous System, 9, 7991. http://dx.doi.org/10.1111/j.1085-9489.2004.009206.x

[14] Leger, J.M., Younes-Chennoufi, A..B., Zuber, M., Bounche, P., Jauberteau, M.O., Dormont, D., Danton, F., Baumann, N. and Brunet, P. (1992) Frequency of central lesion in polyneuropathy associated with IgM monoclonal gammopathy: An MRI, neurophysiological and immunochemical study. Journal of Neurology, Neurosurgery \& Psychiatry, 55, 112-115. http://dx.doi.org/10.1136/jnnp.55.2.112

[15] Barbieri, S., Nobile-Orazio, E., Baldini, L., Fayoumi, Z.,
Manfredini, KE. and Scarlato, G. (1997) Visual evoked potentials with neuropathy and macroglobulinemia. Annals of Neurology, 22, 663-666. http://dx.doi.org/10.1002/ana.410220520

[16] Quarles, R.H. (2007) Myelin-associated glycoprotein (MAG): Past, present and beyond. Journal of Neurochemistry, 100, 1431-1438.

[17] Fluri, F., Ferracin, F., Beat, E. and Steck, A.J. (2003) Microheterogeneity of anti-myelin-associated glycoprotein antibodies. Journal of the Neurological Sciences, 207, 43-49. http://dx.doi.org/10.1016/S0022-510X(02)00359-3

[18] Di Trapani, G., Carnevale, A., Cioffi, R.P., Massaro, A.R. and Profice, P. (1996) Multiple Sclerosis associated with peripheral demyelinating neuropathy. Clinical Neuropathology, 15, 135-138.

[19] Drulovic, D.S., Levic, Z., Stoiasavljevic, N., Trikic, R., Cvetkovic, D. and Apostolski, S. (1998) Unusual association of multiple sclerosis and tomaculous neuropathy. Journal of the Neurological Sciences, 157, 217-222. http://dx.doi.org/10.1016/S0022-510X(98)00054-9

[20] Falcone, M., Scalise, A., Minisci, C., Romito, D., Cancelli, I. and Gigli, G.L. (2006) Spreading of autoimmunity from central to peripheral myelin: two cases of clinical association between multiple sclerosis and chronic inflammatory demyelinating polineuropathy. Neurological Sciences, 27, 58-62. http://dx.doi.org/10.1007/s10072-006-0565-3

[21] Pirko, I., Kuntz, N.L., Patterson, M., Keegan, B.M., Weinshenker, B.G. and Rodriguez, M. (2003) Contrasting effects of INF beta and IVIG in children with central and peripheral demyelination. Neurology, 60, 1697-1699. http://dx.doi.org/10.1212/01.WNL.0000064163.94122.E B

[22] Pogorzelski, R., Baniukiewicz, E. and Drozdowski, W. (2004) Subclinical lesions of peripheral nervous system in multiple sclerosis patients. Neurologia i Neurochirurgia Polska, 38, 257-264.

[23] Quan, D., Pelak, V., Tanabe, J., Duraira, V. and Kleinschmidt-Demasters, B.K. (2005) Spinal and cranial hypertrophic neuropathy in multiple sclerosis. Muscle Nerve, 31, 772-779. http://dx.doi.org/10.1002/mus.20312

[24] Roy, Y.I., Alexander, C.B. and Oh, S.J. (1983) Multiple sclerosis and hypertrophic demyelinating peripheral neuropathy. Muscle Nerve, 6, 312-316. http://dx.doi.org/10.1002/mus.880060411

[25] Tachi, N., Ishikawa, Y., Tsuzuki, A., Minami, R., Sasaki, K. and Shinoda, M. (1985) A case of childhood multiple sclerosis with peripheral neuropathy. Neuropediatrics, 16, 231-234. http://dx.doi.org/10.1055/s-2008-1059543

[26] Laura, M., Leong, W., Murray, M.F., Ingle, G., Miszkiel, K.A., Altmann, D.R., Miller, D.H. and Reilly, M.M. (2005) Chronic inflammatory demyelinating polyradiculoneuropathy: MRI study of brain and spinal cord. $\mathrm{Neu}$ rology, 64, 914-916.

http://dx.doi.org/10.1212/01.WNL.0000152842.11864.D0

[27] Nadkarni, N. and Lisak, R.P. (1993) GuillanBarrè syndrome (GBS) with bilateral optic neuritis and central white matter disease. Neurology, 43, 843-842. http://dx.doi.org/10.1212/WNL.43.4.842 
[28] Pinedda, A.A., Ogata, K., Osoegawa, M., Murai, H., Shigeto, H., Yoshiura, T., Tobimatsu, S. and Kira, J. (2007) A distinct subgroup of chronic inflammatory demyelinating polineuropathy with CNS demyelination and favoreble response to immunotherapy. Journal of the Neurological Sciences, 255, 1-6. http://dx.doi.org/10.1016/j.jns.2007.01.004

[29] de Lau, L.M.I. and Breteler, M.M.B. (2006) Epidemiology of Parkinson's disease. The Lancet Neurology, 5, 525-535.

http://dx.doi.org/10.1016/S1474-4422(06)70471-9

[30] Mariotti, C., Gellero, C., Rimoldi, M., Mineri, R., Uziel, G., Zorzi, G., Pareyson, D., Piccolo, G., Gambi, D., Piacentini, S., Squitieri, F., Capra, R., Castelletti, B. and Di Donato, S. (2004) Ataxia with isolatedvit. Edeficiency: neurological phenotype, clinical follow-up and novel mutations in TTPA gene in Italian families. Neurological Sciences, 25, 130-137. http://dx.doi.org/10.1007/s10072-004-0246-z

[31] Dispenzieri, A. and Kyle, R.A. (2005) Neurological aspects of multiple myeloma and related disorders. Best Practice \& Research Clinical Haematology, 18, 673-688. http://dx.doi.org/10.1016/j.beha.2005.01.024

[32] Bida, J.P, Kyle, R.A., Therneau, T.M., et al. (2009) Disease associations with monoclonal gammopathy of undetermined significance: A population-based study of 17.398 patients. Mayo Clinic Proceedings, 84, 685-693. http://dx.doi.org/10.4065/84.8.685 